\title{
Adaptive Hysteresis Compensation with a Dynamic Hysteresis Model for Control of a Pneumatic Muscle Actuator*
}

\author{
Takahiro KOSAKI**, Ayuto MINESAKI** and Manabu SANO** \\ ** Department of Systems Engineering, Hiroshima City University \\ 3-4-1 Ozuka-higashi, Asaminami-ku, Hiroshima 731-3194, Japan \\ E-mail: kosaki@ hiroshima-cu.ac.jp
}

\begin{abstract}
This paper proposes a technique for modeling the dynamic behavior of the hysteresis in pneumatic muscle actuators (PMAs) and controlling the motion of PMAs with the hysteresis model. PMAs contract according to the internal pressure level; however, the relationship between the contraction and the internal pressure is subject to hysteresis, and the hysteresis distorts depending on the acting speed of PMAs. To describe such hysteresis, the proposed technique uses the dynamic Preisach model and adaptively tunes the parameters of the model by recursive parameter estimation if the distortion occurs due to speed variations. The hysteresis model with the on-line adjusted parameters was incorporated into a PMA control system as the adaptive hysteresis compensator for the purpose of reducing undesirable effects caused by the hysteresis on the control accuracy. Additionally, as introducing a fast inversion algorithm enabled the real-time calculation of hysteresis compensator outputs, the hysteresis compensator could cope with the reference-input changes while the system was in operation. Experimental results showed that the proposed technique achieved higher tracking performance than the technique developed in our previous work, both for a single PMA and for a parallel manipulator driven by three PMAs.
\end{abstract}

Key words : Oil and Air Hydraulics, Pneumatic Muscle Actuator, Motion Control, Hysteresis, Modeling

\section{Introduction}

Institutions providing medical and social-welfare services face labor shortages in the future, due to the aging of many advanced societies. The hope is that robots will be able to play a role in filling this gap. Such assistive robots must, in the first place, be safe to use in a human environment, especially one populated by the frail elderly. Pneumatic muscle actuators (PMAs) ${ }^{(1)}$ are suitable for driving those types of robots because they are lightweight and highly flexible and would not hurt a person even in a collision ${ }^{(2),(3)}$. A PMA consists of a flexible rubber tube surrounded by a braided-fiber shell. Due to the elasticity of the fibers, the frictional loss between the rubber tube and the fibers, and the elasticity of the rubber, the relationship between the contraction and the internal pressure in the pressurizing process differs from that in the depressurizing process. The difference in the relationship causes hysteresis nonlinearity, which often leads to difficulty in modeling PMAs and inaccuracy in controlling them. Several approaches to deal with such hysteresis have been proposed up to now ${ }^{(4)}$, but a fully satisfactory one does not seem to be established. We previously showed that the Preisach model $^{(5)}$ for magnetic materials is applicable for modeling the hysteresis of a PMA and that incorporating a feedforward compensator with the hysteresis model into the control system of the PMA can improve the tracking performance to some extent ${ }^{(6)}$. However, the following two drawbacks relating to the hysteresis model were present in this technique:

( 1 ) Hysteresis curves in PMAs usually distort according to increases in their contracting

*Received 14 May, 2012 (No. 12-0153) [DOI: 10.1299/jee.7.53]

Copyright (c) 2012 by JSME 
and expanding speed. The hysteresis compensator in our previous technique is based on a hysteresis model identified from the preliminarily measured characteristics of a PMA and does not have any adjustable parameters. Thus, at higher contracting and expanding speeds, the gap between the actual hysteresis and its model increases, and that gives rise to the trackingperformance deterioration in the control of the PMA.

( 2 ) Due to the use of an iterative algorithm, the computational complexity for obtaining the hysteresis compensator outputs is too large for real-time calculation.

The objective of this study is to propose a technique to overcome these drawbacks and to improve the control performance. To cope with the former issue, we model a PMA in the form of the dynamic Preisach model ${ }^{(5)}$. In addition, the parameters included in the model are adjusted by recursive parameter estimation ${ }^{(8)}$. To manage the latter issue, we employ a fast algorithm $^{(10)}$ for finding the inverse of the Preisach model. The expected advantages of the present technique can be summarized as follows:

- The dynamic Preisach model can be constructed from the same quantity of measured data as required in the static Preisach model, though it has parameters requiring on-line adjustment.

- The performance of the designed control system depends on the accuracy of the hysteresis model of a PMA. Using a dynamic hysteresis model with the adaptively tuned parameters leads to the reduction of the modeling errors caused when the contracting or expanding speed of a PMA increases, without any manual tuning.

- The fast inversion algorithm provides a hysteresis compensator with the real-time processing. Therefore, the hysteresis compensator is able to cope with the reference-input alterations while the system itself is in operation.

Based on the proposed technique, we build control systems for a single PMA and for a parallel manipulator driven by three PMAs (PMPMA) and experimentally verify their performance.

The remainder of this paper is organized as follows: Section 2 describes the details of the dynamic Preisach model and modeling experiment results. Section 3 gives the configuration of the control system for the PMA that we propose and the experimental results. Section 4 presents the control system for the PMPMA and the results of verification experiments. Section 5 provides conclusions.

\section{Hysteresis modeling}

\subsection{Dynamic hysteresis model}

Although our former paper ${ }^{(6)}$ suggested using the Preisach model to describe the hysteresis nonlinearity in PMAs, its inability to describe the dynamic behavior of the hysteresis often causes modeling errors. In this paper, we introduce a dynamic Preisach model and apply it to model PMAs.

The mathematical form of the Preisach model is generally defined as follows ${ }^{(5)}$ :

$$
f(t)=\iint_{\alpha \geq \beta} \mu(\alpha, \beta) \gamma_{\alpha \beta}[u(t)] d \alpha d \beta
$$

where $f(t)$ is the output, $u(t)$ is the input, $\gamma_{\alpha \beta}$ denotes a hysteresis operator that outputs 1 when $u(t) \geq \alpha$ and -1 when $u(t) \leq \beta$, and $\mu(\alpha, \beta)$ is a weight function that expresses the distribution of $\gamma_{\alpha \beta}$, which is called the Preisach function. The model (1) is hereafter referred to as the "static" Preisach model.

To represent the dynamic behavior of hysteresis, a dynamic Preisach model also exists ${ }^{(5)}$. The main idea behind this model is to introduce the dependence of the Preisach function on the speed of output variations, $\frac{d f}{d t}$; i.e., $\mu(\alpha, \beta)$ in Eq. (1) is replaced by $\mu\left(\alpha, \beta, \frac{d f}{d t}\right)$. To avoid the difficulty in the identification of $\mu\left(\alpha, \beta, \frac{d f}{d t}\right)$, the power series expansions for $\mu\left(\alpha, \beta, \frac{d f}{d t}\right)$ with respect to $\frac{d f}{d t}$ are usually used:

$$
\mu\left(\alpha, \beta, \frac{d f}{d t}\right)=\mu_{0}(\alpha, \beta)+\frac{d f}{d t} \mu_{1}(\alpha, \beta)+\cdots .
$$


By retaining the first two terms of Eq. (2), we can obtain the following first-order dynamic model:

$$
f(t)=\iint_{\alpha \geq \beta} \mu_{0}(\alpha, \beta) \gamma_{\alpha \beta}[u(t)] d \alpha d \beta+\frac{d f}{d t} \iint_{\alpha \geq \beta} \mu_{1}(\alpha, \beta) \gamma_{\alpha \beta}[u(t)] d \alpha d \beta .
$$

Since Eq. (3) should coincide with Eq. (1) if $\frac{d f}{d t}=0$, the first term of Eq. (3) means the static component of hysteresis; i.e., $\mu_{0}$ is equivalent to $\mu$ in Eq. (1). We represent the first static term as $\tilde{f}(t)$. The function $\mu_{1}$ in the second term of Eq. (3) is usually determined by fitting the model to experimental data. Thus, we can represent the second term as $\tau(t) \frac{d f}{d t}$ with the time-varying parameter $\tau(t)$ to be acquired experimentally. As a result, the model (3) can be rewritten as the following differential equation:

$$
\tau(t) \frac{d f}{d t}+f(t)=\tilde{f}(t)
$$

Similarly, using the first three terms of Eq. (2) leads finally to the following second-order dynamic model:

$$
\tau_{1}(t) \frac{d^{2} f}{d t^{2}}+\tau_{2}(t) \frac{d f}{d t}+f(t)=\tilde{f}(t) .
$$

$\tau_{1}(t)$ and $\tau_{2}(t)$ are also determined with measured data.

\subsection{Adaptive tuning of model parameters}

Iyer and $\operatorname{Tan}^{(7)}$ presented adaptive identification of the static Preisach model (1). Their method requires the reconstruction of Eq. (1) whenever the hysteresis-curve distortion occurs and takes much time to identify Eq. (1) accurately. Meanwhile, the procedure described in Ref. (5) to find $\tau(t)$ in Eq. (4) or $\tau_{1}(t)$ and $\tau_{2}(t)$ in Eq. (5) from experimental data is not adaptive. Hence, we propose to cope with the hysteresis-curve distortion in PMAs by utilizing the dynamic Preisach model with the off-line identified Eq. (1) and the adaptively tuned parameters.

By approximating Eqs. (4) and (5) with the backward difference, we obtain

$$
f(k)=-\left(\frac{h}{\tau}-1\right) f(k-1)+\frac{h}{\tau} \tilde{f}(k-1)
$$

and

$$
f(k)=-\left(\frac{\tau_{1}}{\tau_{2}} h-2\right) f(k-1)-\left(\frac{h^{2}}{\tau_{2}}-\frac{\tau_{1}}{\tau_{2}} h+1\right) f(k-2)+\frac{h^{2}}{\tau_{2}} \tilde{f}(k-2)
$$

where $h$ is the sampling period and $k$ is the discrete time-step. These equations correspond to typical linear regression model structure, i.e., $f(k)=\boldsymbol{\Theta}^{T} \boldsymbol{\Phi}$, where $\boldsymbol{\Theta}$ is the parameter vector and $\boldsymbol{\Phi}$ is the regression vector. In addition, since $\tau(t), \tau_{1}(t)$, and $\tau_{2}(t)$ are time-varying parameters, adaptive methods have to be used to track their variations. In order to find these parameters, we employed the following recursive parameter estimation ${ }^{(8)}$ for linear regression models based on the least mean square method:

$$
\hat{\boldsymbol{\Theta}}(k)=\hat{\boldsymbol{\Theta}}(k-1)+\lambda \frac{\boldsymbol{\Phi}(k)}{\boldsymbol{\Phi}^{T}(k) \boldsymbol{\Phi}(k)} \epsilon(k),
$$

where $\lambda$ is the step size parameter, $\epsilon(k)$ is the error between the desired value and the estimate. The estimate converges if $0<\lambda<2^{(9)}$. This method is widely used in the field of adaptive digital signal processing because of quick convergence and easy incorporation into a computer. For the first-order dynamic Preisach model (4), we defined $\boldsymbol{\Theta}$ as $\left[\frac{h}{\tau}-1, \frac{h}{\tau}\right]^{T}$ and $\boldsymbol{\Phi}$ as $[-f(k-1), \tilde{f}(k-1)]^{T}$, and for the second-order dynamic Preisach model (5), we defined $\boldsymbol{\Theta}$ as $\left[\frac{\tau_{1}}{\tau_{2}} h-2, \frac{h^{2}}{\tau_{2}}-\frac{\tau_{1}}{\tau_{2}} h+1, \frac{h^{2}}{\tau_{2}}\right]^{T}$ and $\boldsymbol{\Phi}$ as $[-f(k-1),-f(k-2), \tilde{f}(k-2)]^{T}$. These vectors are based on Eqs. (6) and (7). By substituting the difference between the actual contraction of a PMA and the model output into $\epsilon$ in Eq. (8), the model parameters can be adjusted on-line. 


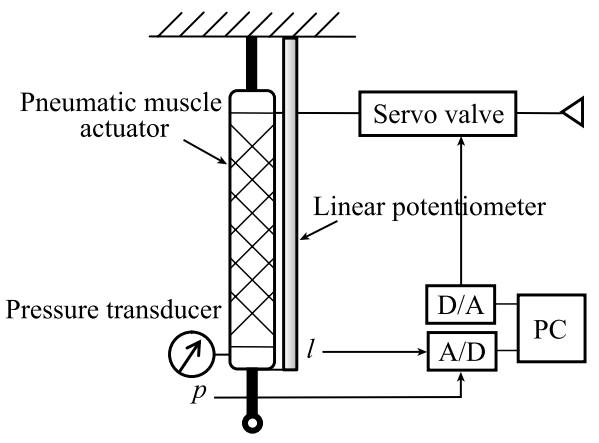

Fig. 1 Schematic of the experimental setup for a pneumatic muscle actuator (PMA)

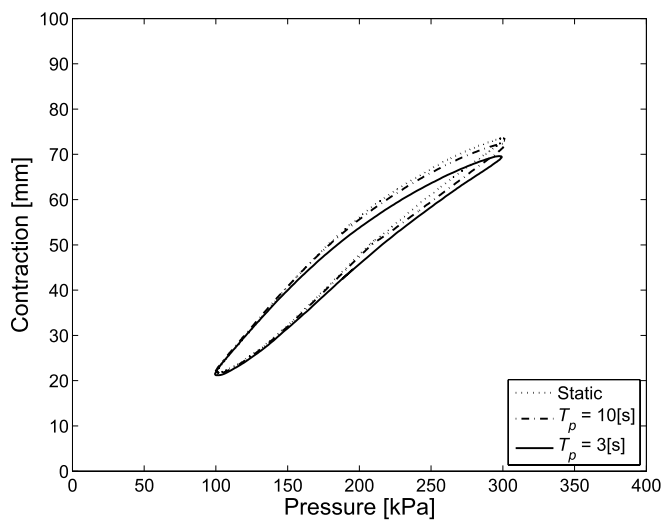

Fig. 2 Measured hysteresis examples in the PMA

\subsection{Modeling experiment}

2.3.1. Experimental setup We constructed a dynamic Preisach model of a PMA and conducted experiments to evaluate the modeling accuracy. Figure 1 illustrates the schematic of the experimental setup used for the modeling and the evaluation. The investigated PMA has a diameter of $20 \mathrm{~mm}$ and a nominal length of $400 \mathrm{~mm}$. The internal pressure $p$ is detected by a pressure transducer, and its signal is sent to a PC through an A/D interface. A PID control algorithm for regulating the internal pressure is implemented on the PC, which produces the operating voltage of a proportional control valve to adjust the air flow rate into the actuator. Applying the voltage through a D/A interface to the valve allows the internal pressure to be set to a reference pressure. The contraction of the PMA, $l$, is measured by a linear potentiometer. The outputs of all the sensing devices are recorded on the PC.

The contraction-pressure curves of the PMA measured using this experimental setup are shown in Fig. 2. In the measurements, sine signals with an amplitude of $100 \mathrm{kPa}$, a bias pressure of $200 \mathrm{kPa}$, and time periods $T_{p}$ of 3,10 , and $60 \mathrm{~s}$ were inputted to the pressure controller as the reference inputs (we here consider the 60-s case to be static). As seen in Fig. 2 , hysteresis appears and distorts gradually in accordance with the increase of the contracting and expanding speed.

2.3.2. Modeling results As stated in $\S 2.1$, the dynamic Preisach model consists of the static Preisach model (1) (i.e., $\tilde{f}(t)=f(t)$ in Eq. (1)) and Eq. (4) for the first-order model or Eq. (5) for the second-order model. We defined the contraction and the internal pressure of the PMA as the output $f(t)$ and the input $u(t)$ in these equations, respectively. Consequently, we stipulated that the output of the hysteresis operator $\gamma_{\alpha \beta}$ has two values, 0 and 1, as shown in Fig. 3 because the contraction and the internal pressure are always positive. In this case, since there is a one-to-one correspondence between $\gamma_{\alpha \beta}$ and points $(\alpha, \beta)$ on the $\alpha-\beta$ plane from the geometrical viewpoint, the output of the static Preisach model depends only on subdivision corresponding to $\alpha \geq \beta$ and $\gamma_{\alpha \beta}=1$ on the $\alpha-\beta$ plane (e.g., Fig. 4).

We first have to identify the Preisach function $\mu(\alpha, \beta)$ in the static Preisach model (1) 


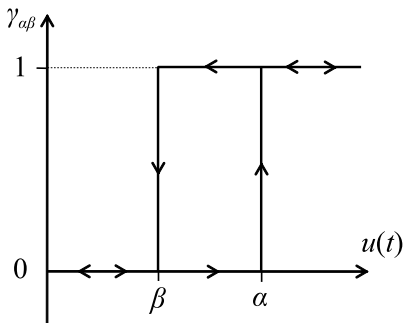

Fig. 3 Hysteresis operator

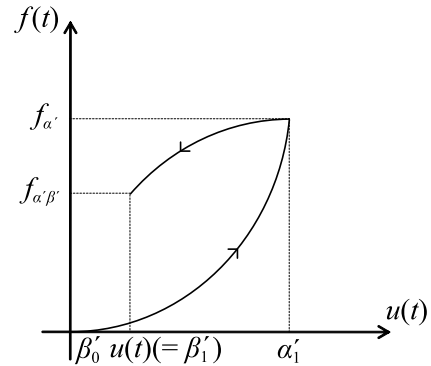

(a) A hysteresis curve

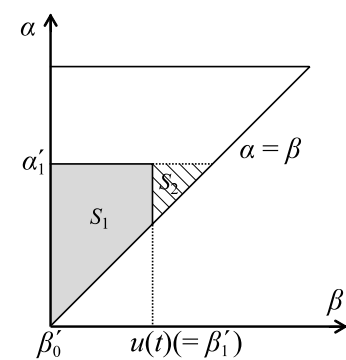

(b) The $\alpha-\beta$ diagram corresponding to (a)

Fig. 4 Geometric interpretation of the static Preisach model

from the measured data. To facilitate this identification, the following function $F\left(\alpha^{\prime}, \beta^{\prime}\right)$ based on the geometrical mapping of a variation on the input-output curve (see Fig. 4 (a)) to a variation $S_{2}$ of subdivision $S_{1}$ on the $\alpha-\beta$ plane (see Fig. 4 (b)) is generally employed ${ }^{(5)}$; i.e., on the $\alpha-\beta$ plane,

$$
F\left(\alpha^{\prime}, \beta^{\prime}\right)=\iint_{S_{2}\left(\alpha^{\prime}, \beta^{\prime}\right)} \mu(\alpha, \beta) d \alpha d \beta
$$

while on the output-input plane, $F\left(\alpha^{\prime}, \beta^{\prime}\right)=f_{\alpha^{\prime}}-f_{\alpha^{\prime} \beta^{\prime}}$, where $f_{\alpha^{\prime}}$ is the output on an ascending curve when $u(t)=\alpha^{\prime}$ and $f_{\alpha^{\prime} \beta^{\prime}}$ is the output on a descending curve from $\alpha^{\prime}$ when $u(t)=\beta^{\prime}$. For determining the above $F\left(\alpha^{\prime}, \beta^{\prime}\right)$, we extracted $f_{\alpha^{\prime}}$ and $f_{\alpha^{\prime} \beta^{\prime}}$ from experimental data collected by inputting sinusoidal reference inputs with a time period of $60 \mathrm{~s}$, a bias pressure of $250 \mathrm{kPa}$, and an amplitude from $0 \mathrm{kPa}$ to $250 \mathrm{kPa}$ to the pressure controller. The data were recorded under a supply pressure of $560 \mathrm{kPa}$ at a sampling time of $5 \mathrm{~ms}$. In the extraction process of $f_{\alpha^{\prime}}$ and $f_{\alpha^{\prime} \beta^{\prime}}$, the discretization level on the $\alpha-\beta$ plane was set as 40 for each axis. It is possible to directly find the output of the static Preisach model by means of the identified function $F\left(\alpha^{\prime}, \beta^{\prime}\right)$ (we use interpolation to compute the output at points where measured data does not exist). The details are given in Ref. (6).

Figure 5 shows modeling result examples for a sinusoidal input with a time period of $3 \mathrm{~s}$, an amplitude of $100 \mathrm{kPa}$, and a bias pressure of $200 \mathrm{kPa}$. In Fig. 5, the dotted line indicates the measured hysteresis, and the dashed line indicates the result simulated with the static Preisach model. The result simulated with the first-order dynamic Preisach model and the recursive parameter estimation and that with the second-order dynamic Preisach model and the recursive parameter estimation are plotted by the dash-dotted line and the solid line, respectively. Taking account of the convergence condition, we chose $\lambda=0.02$ for the first-order model and $\lambda=0.1$ for the second-order model by a trial-and-error approach. The initial values in the recursive parameter estimation were roughly determined in advance. To quantify the modeling accuracy, the RMSEs (root mean square errors) between the measured and simulated outputs were calculated with the static, the first-order dynamic, and the second-order dynamic Preisach models for sine inputs with time periods of 2, 3, 4, 6, and $8 \mathrm{~s}$. The RMSEs on average were 0.49 for the static model, 0.062 for the first-order model, and 0.056 for the second-order model, respectively. From these errors and Fig. 5, it was confirmed that the dynamic models could precisely represent the hysteresis in the high-speed motion of the PMA, while the static 


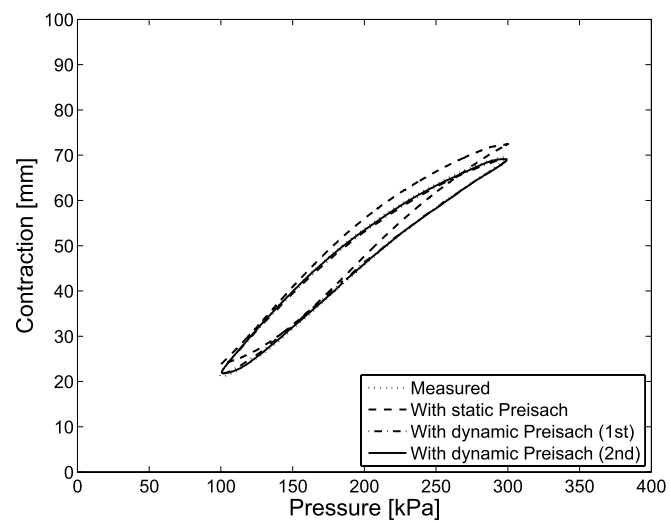

Fig. 5 Modeling results of the PMA with the static and dynamic Preisach models

model could not. In addition, the RMSE for the second-order model is smaller by about $10 \%$ than that for the first-order model.

\section{Hysteresis compensation control of a PMA}

\subsection{PMA control system}

The dynamic Preisach model was utilized for controlling the motion of a PMA. Figure 6 (a) illustrates the structure of the control system that we propose. In the figure, $l$ is the contraction of the PMA, $p$ is the internal pressure, $p_{f}$ is the hysteresis compensator output, $v$ is the operating voltage of the control valve, and the subscript $d$ denotes the reference. Although it is apparent that the dynamic Preisach model has to be adjusted based on the modeling error between the model output $l_{m}$ and the actual contraction $l$ as shown in Fig. 6 (b), such a structure increases the computational complexity of the control algorithm and needs greater effort to incorporate the control algorithm into a computer, in comparison with the simplified structure given in Fig. 6 (a). Therefore, we adopted the simplified control system. The influence of the simplification on the control performance is discussed later. The basic part of this control system is the same as that used in Ref. (6), which is composed of feedback and feedforward components. The feedback component comprises an inner PID pressure-control loop and an outer PID contraction-control loop (the pressure and contraction control laws are given in Ref. (6)). The inner loop is introduced to reduce the time delay of the internal pressure change in response to the control command signal, because the motion of the PMA depends greatly on the responsiveness of the internal pressure. The role of the feedforward component, namely the hysteresis compensator, is to reduce the undesirable hysteresis effect. The present control system differs from our former control system ${ }^{(6)}$ in the following ways:

- The hysteresis compensator produces $p_{f}$ based on the inverse of the dynamic Preisach model. In the case of the first-order model, the inverse of Eq. (6) is represented by

$$
\tilde{f}(k)=\frac{\tau}{h} f(k+1)+\left(1-\frac{\tau}{h}\right) f(k)
$$

while in the second-order model case, that of Eq. (7) is represented by

$$
\tilde{f}(k)=\frac{\tau_{2}}{h^{2}} f(k+2)+\left(\frac{\tau_{1}}{h}-2 \frac{\tau_{2}}{h^{2}}\right) f(k+1)+\left(\frac{\tau_{2}}{h^{2}}-\frac{\tau_{1}}{h}+1\right) f(k),
$$

where $f$ corresponds to the reference input $l_{d}$. The value $\tilde{f}$, obtained by substituting $f$ into Eq. (10) or Eq. (11), is inputted to the inverse of the static Preisach model, and a $p_{f}$ for $\tilde{f}$ is produced. As $f\left(=l_{d}\right)$ is predetermined, we can use its future values.

- The parameters included in the inverse of the dynamic Preisach model (i.e., $\tau, \tau_{1}$, and $\tau_{2}$ ) are adjusted on-line by applying the recursive parameter estimation mentioned in $\$ 2.2$ to Eqs. (10) and (11).

- The hysteresis compensator output $p_{f}$ is calculated in real time by employing the fast inversion algorithm, which is described in the next section.

Accordingly, as the reference $l_{d}$ is inputted into the adaptive part of our control system, the 


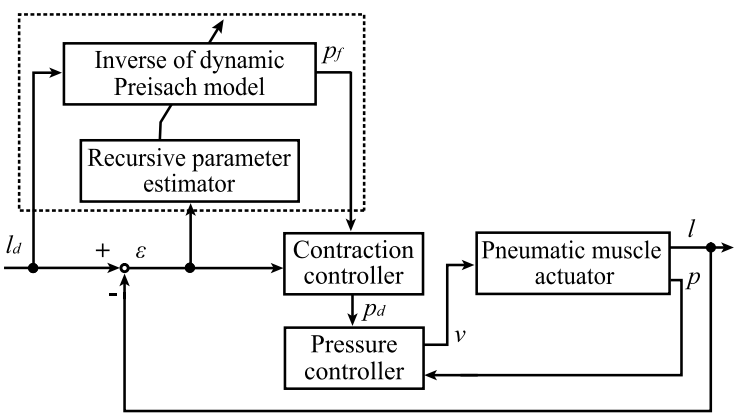

(a) Simplified structure

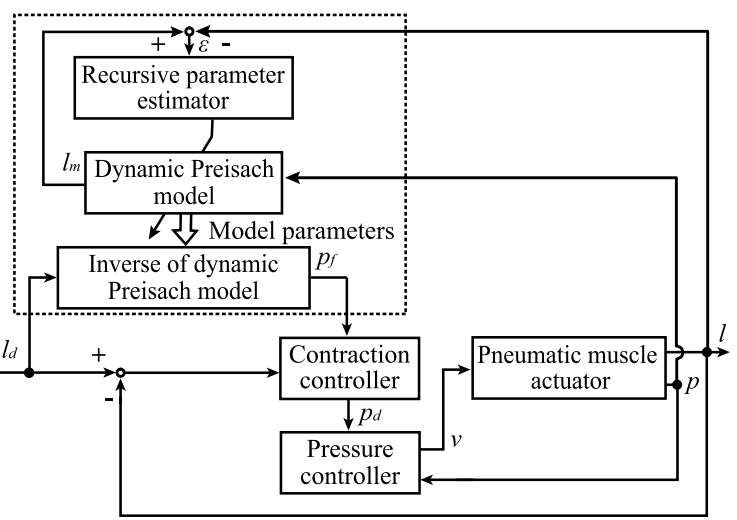

(b) Original structure

Fig. 6 Control system structure for the PMA with the hysteresis compensator based on the dynamic Preisach model

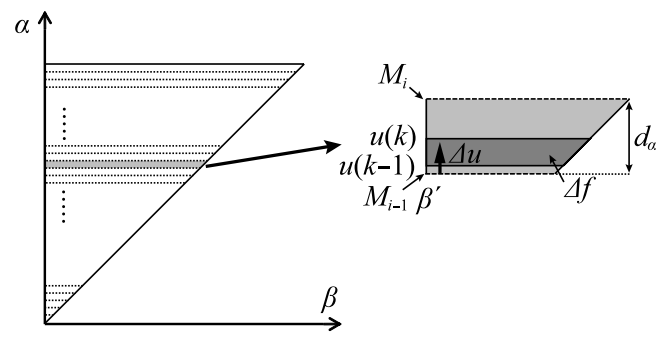

Fig. 7 Fast inversion algorithm for monotonically increasing inputs

difference between the current contraction $l$ and $l_{d}$ is fed into Eq. (8) and $\tau, \tau_{1}$, and $\tau_{2}$ are updated. Simultaneously, Eqs. (10) and (11) with such parameters give $\tilde{f}$ for $l_{d}$. Then, using the static Preisach model obtained in $\$ 2.3 .2$ and the fast inversion algorithm for the static Preisach model, we derive $p_{f}$ for $\tilde{f}$.

\subsection{Fast inversion algorithm}

Our previous technique ${ }^{(6)}$ used an iterative algorithm to find the inverse of the static Preisach model. Since this algorithm takes much time, the hysteresis compensator output for the entire path of the reference input has to be calculated in advance. Hence, we have to recalculate the hysteresis compensator output if the reference input is changed. In the present study, we utilize the fast inversion algorithm developed by Davino et al. ${ }^{(10)}$.

Defining the discretization lattice on the $\alpha-\beta$ plane as shown in Fig. 7, this algorithm yields the inverse of the static Preisach model for monotonically increasing inputs by the following equation:

$$
\begin{aligned}
& u(k)=\frac{d_{\alpha}}{F\left(M_{i}, \beta^{\prime}\right)-F\left(M_{i-1}, \beta^{\prime}\right)} \Delta F+(i-1) d_{\alpha}, \\
& \Delta F=\Delta f+F\left(u(k-1), \beta^{\prime}\right)-F\left(M_{i-1}, \beta^{\prime}\right),
\end{aligned}
$$


where $M_{i}$ is the coordinate of the $i$ th lattice point on the $\alpha$-axis, the distance $d_{\alpha}=M_{i}-M_{i-1}$, $\beta^{\prime}$ is the $\beta$-value of the extremum of the hysteresis curve, $\Delta f$ is the output variation when the input varies from $u(k-1)$ to $u(k)$, and $F$ is given in $\$ 2.3$. This algorithm only uses $F$-function values and does not require any iterations. Here we can consider the meaning of Eq. (12) as follows. For monotonically increasing inputs, defining the inverse $\Gamma_{\alpha}:(F, \beta) \mapsto \alpha$ for $F$, we can represent an unknown input $u$ to be found as $u=\Gamma_{\alpha}(F, \beta)$. By linearizing $\Gamma_{\alpha}$ with respect to $F$ and $\beta$, we get

$$
\Delta u=\frac{\partial \Gamma_{\alpha}}{\partial F} \Delta F+\frac{\partial \Gamma_{\alpha}}{\partial \beta} \Delta \beta .
$$

In the case of monotonic increase, $\Delta \beta=0$ in Eq. (13). The value $u$ can be also regarded as $u=\Delta u+M_{i-1}=\Delta u+(i-1) d_{\alpha}$. Therefore, Eq. (12) is derived by approximately expressing Eq. (13) with $F$. In the case of monotonic decrease, $u$ can be obtained by the same procedure stated above. In the actual calculation of $u(k)$, i.e., the hysteresis compensator output $p_{f}(k)$ at the current step $k$, since $F$ is known (this is obtained in $\$ 2.3 .2$ ), $\Delta f$ is measurable, and a variation from $u(k-1)$ to $u(k)$ is extremely small, we can find $p_{f}(k)$ based on the linear relationship (12).

The actual performance of the fast inversion algorithm was compared with that of the algorithm used in the previous paper ${ }^{(6)}$. Both the algorithms contain the static Preisach model of a PMA constructed in $\$ 2.3$; accordingly, the discretization level on the $\alpha-\beta$ plane is 40 for each axis. These algorithms were implemented on a PC with an Intel Core 2 Duo $3.0 \mathrm{GHz}$ CPU and 4 GB RAM, which is also employed as the PMA controller in the next section. The elapsed time to calculate the inverse output of the static Preisach model throughout each of sinusoidal reference inputs with an amplitude of $35 \mathrm{~mm}$ and time periods of 5, 10, 15, and 20 $\mathrm{s}$ was measured, and then we found the average elapsed time of five measurements for every time period. As a result, the fast inversion algorithm took approximately $0.021 \mathrm{~ms}$ per one process, while the previous algorithm took approximately $17 \mathrm{~ms}$ per one process. The fast inversion algorithm is capable of realizing the real-time calculation of the inverse output of the static Preisach model because the sampling time in the control experiments in the next section is $5 \mathrm{~ms}$.

\subsection{Experimental results}

To test the tracking performance of the present PMA control system, we performed experiments with the apparatus shown in Fig. 1. Our previously proposed control system ${ }^{(6)}$ with the static Preisach model was also examined for comparison. The values of the gains in the feedback controller were determined by a trial-and-error approach under the consideration of sensitivity to noise, but the same values were used in the two systems. When the PMA was pressurized initially up to the bias pressure $p_{0}(=285 \mathrm{kPa})$, we defined it as the equilibrium state. The initial values in the recursive parameter estimation were fixed in the experiments.

3.3.1. Experiment on system simplification As described in $\$ 3.1$, the control system shown in Fig. 6 (a) is a simplified version of the control system shown in Fig. 6 (b). In the first place, we experimentally confirmed the influence of the simplification on the control performance. For the confirmation, time responses of the motion of the PMA for sinusoidal reference signals with an amplitude of $35 \mathrm{~mm}$ and time periods of 2, 4, 6, and $8 \mathrm{~s}$ were acquired, and then the RMSE between the reference and the actual contraction of the PMA for each time response was found, where the second-order dynamic Preisach model was used. Five data were collected for every time period. The resulting average RMSE for all the data in the simplified control system was higher by about $2 \%$ than that in the control system without the simplification (=0.013). Accordingly, we neglected the influence of the simplification on the control performance and used only the simplified control system below.

3.3.2. Experiment on tracking performance This test aims at evaluating the tracking performance by quantifying it with the RMSE between the reference input and the actual contraction of the PMA. Sine signals with amplitudes $A$ of 25 and $35 \mathrm{~mm}$ and time periods of 


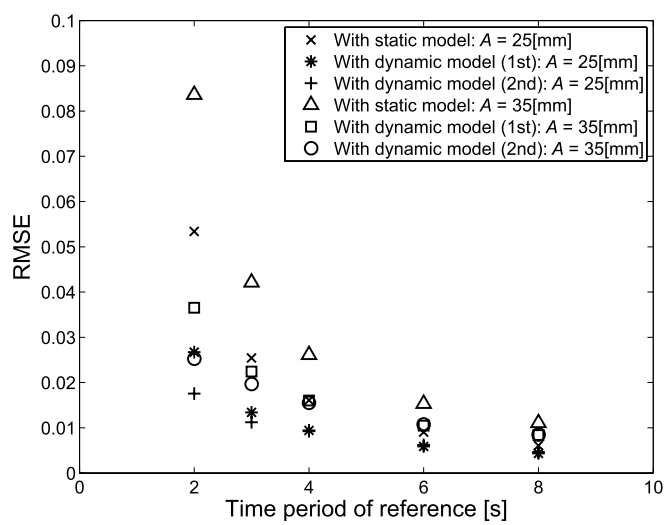

Fig. 8 Transition of the RMSE for changing the time period of the reference in the PMA control
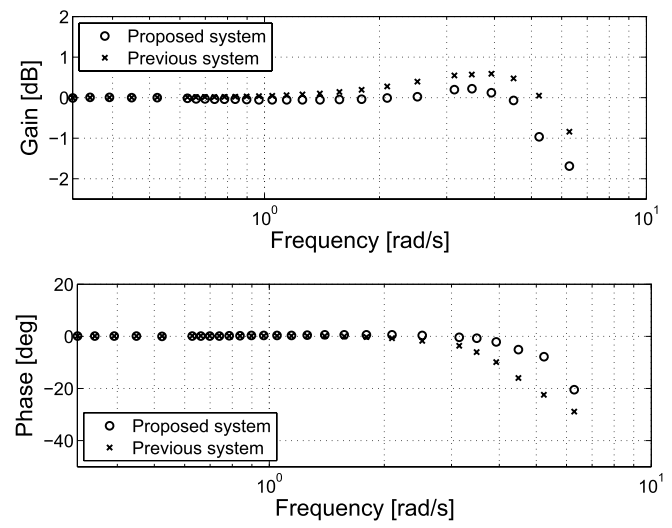

Fig. 9 Comparison of experimental frequency responses in the proposed and previous PMA control systems

$2,3,4,6$, and $8 \mathrm{~s}$ were used as the reference inputs. For comparison, we investigated not only the proposed control systems with the first- and second-order dynamic Preisach models but also our previous control system with the static Preisach model. The hysteresis compensator outputs were calculated in advance for all the references in the previous system, but the proposed systems do not require such preliminary calculation owing to the fast inversion algorithm. In addition, the proposed ones have a recursive parameter estimator. Figure 8 shows the transition of the RMSEs for increasing the time period of the reference. Every value is the average of two measurements. As shown in Fig. 8, utilizing the proposed systems with the first- and second-order models resulted in greater reduction in the RMSE than in the previous system; moreover, the second-order model achieved the smallest RMSE, especially when the PMA acted very fast. Thus, we focused on the system with the second-order dynamic Preisach model in the subsequent experiments.

3.3.3. Experiment on frequency characteristics Figure 9 plots the frequency responses for the proposed control system and for the previous control system, obtained from the time responses when various sine references with an amplitude of $35 \mathrm{~mm}$ and a time period between $1 \mathrm{~s}$ and $20 \mathrm{~s}$ were inputted. The symbols $\bigcirc$ and $\times$ in this figure denote the experimental data for the former and the latter, respectively. Each value is the average of four measurements. In Fig. 9, the proposed system exhibited better gain and phase performance; the performance improvement in the high-frequency range owes to the use of the dynamic hysteresis model and the adaptive tuning of the model.

3.3.4. Experiment on on-line processing We further examined the competence of the on-line tuning and calculation of the hysteresis compensator in the proposed control system for the following two patterns: a decreasing pattern in which the time period $T_{p}$ of a sinusoidal reference input successively decreases, i.e., as $T_{p}=9 \rightarrow 6 \rightarrow 3 \mathrm{~s}$, and the other is a increasing 


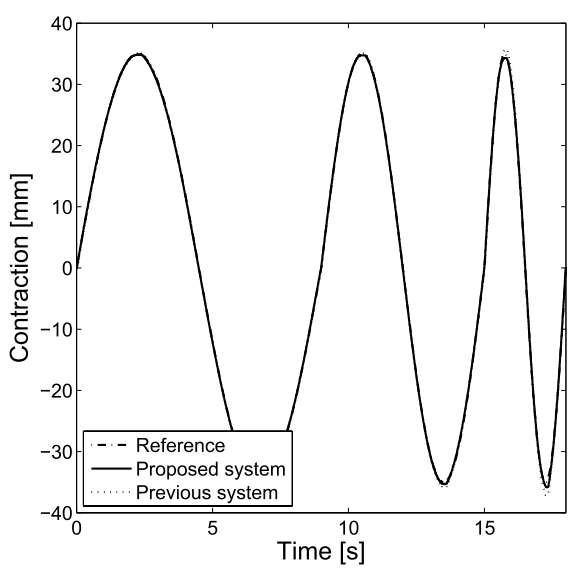

(a) Responses for the decreasing pattern

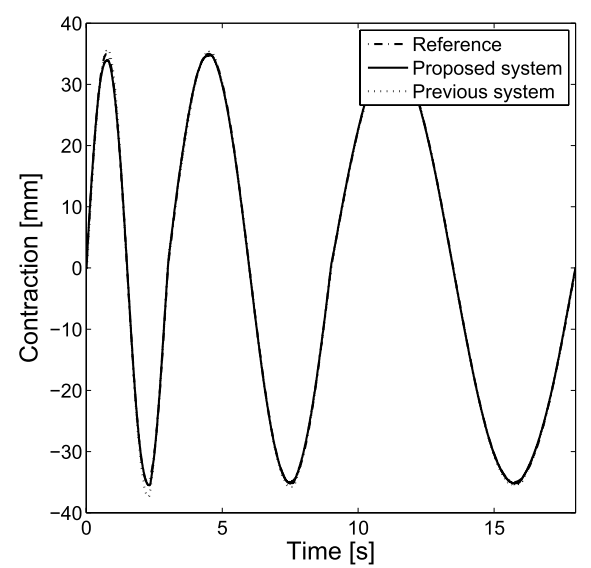

(b) Responses for the increasing pattern

Fig. 10 Comparison of experimental time responses in the proposed and previous PMA control systems

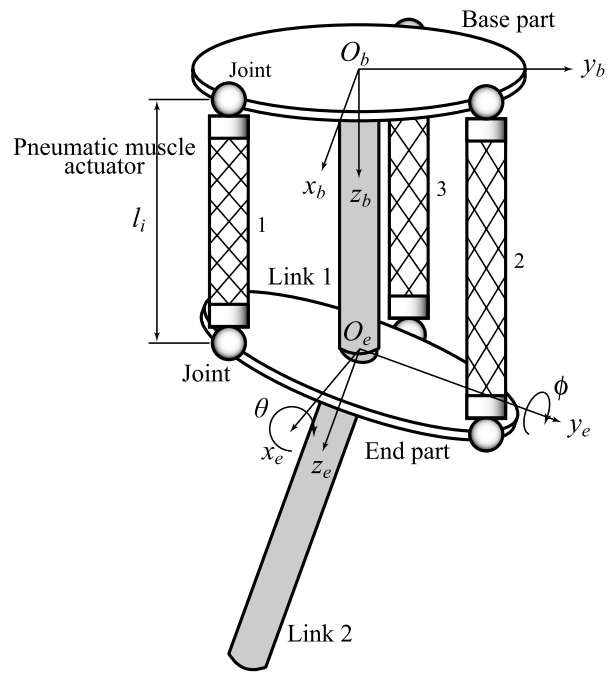

Fig. 11 Schematic of a parallel manipulator driven by three PMAs (PMPMA)

pattern in which $T_{p}$ increases, i.e., $T_{p}=3 \rightarrow 6 \rightarrow 9 \mathrm{~s}$. For both the proposed system and the previous system, the experiments were repeated ten times for each pattern, and then the RMSEs were found. The time response examples are shown in Fig. 10 for the two patterns: (a) the decreasing pattern and (b) the increasing pattern. In these figures, the dashed-dotted lines indicate the references, and the solid and dotted lines indicate results for the proposed system and the previous system, respectively. The average RMSE for the proposed system was 0.0079 and that for the previous system was 0.015. Less overshoot in Fig. 10 and a smaller RMSE were obtained with the proposed system. These results clearly demonstrate that the hysteresis compensator equipped with the fast inversion algorithm worked properly without any manual tuning for the reference variations. Additionally, the proposed technique does not require information about the entire reference path beforehand, though it needs the future reference values up to two subsequent discrete time-steps.

\section{Hysteresis compensation control of a PMPMA}

\subsection{PMPMA}

Further verification of the proposed technique was carried out for the control of a PMPMA. Figure 11 is the schematic of the PMPMA. The coordinate systems $O_{b}-x_{b} y_{b} z_{b}$ and $O_{e}-x_{e} y_{e} z_{e}$ shown in Fig. 11 are referred to hereafter as the base coordinate system and the end coordinate system, respectively. As in the figure, three PMAs connect the movable end part with the fixed base part in parallel through universal joints, and a parallel mechanism is formed. Link 


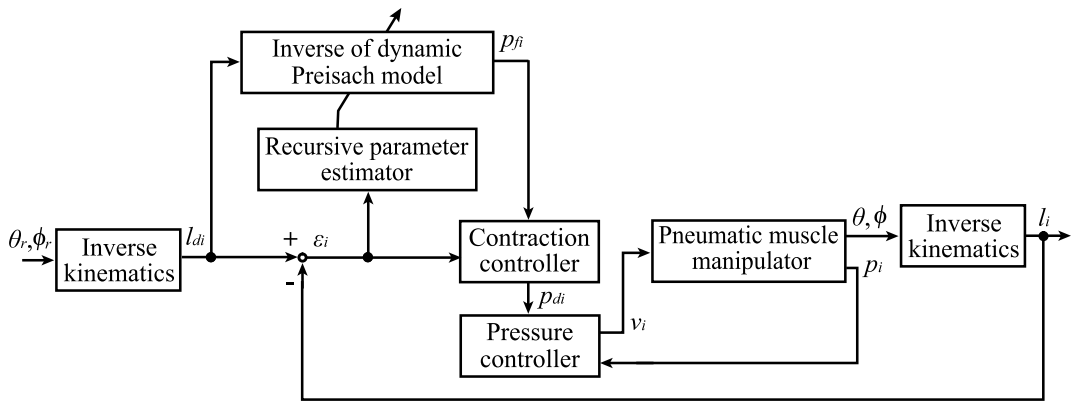

Fig. 12 Structure of the control system for the PMPMA

1 is attached to the center of the base part; only its length is freely adjustable. Link 2 is fixed at the center of the end part. Our original universal joint was employed for the connection between link 1 and link 2 . In this study, the rotation around the $x_{b}$-axis $(\theta)$ and the rotation around the $y_{b}$-axis $(\phi)$ at the origin $O_{e}$ to the base coordinate system are controlled, and the position in the $z_{b}$-direction is always constant. All the PMAs have the same specification as that of the PMA used in the previous section. The radius of the base part and that of the end part are $67 \mathrm{~mm}$, the total length of the actuator with the joints mounted at both ends is 620 $\mathrm{mm}$ when unpressurized, and the length of link 2 is $210 \mathrm{~mm}$. A proportional control valve is connected to a port of each PMA, and a pressure transducer is attached to another port to detect the internal pressure. The rotation angles of link $2(\theta$ and $\phi)$, which can be regarded as the elbow joint angles of the manipulator, are measured by two potentiometers mounted on the end part. The operating voltages of the three valves are computed on a PC, based on the detected angles and pressures, by implementing a control algorithm; individually controlling the three PMAs by feeding them into the valves realizes the bending motion of the elbow joint of the manipulator. The manipulator differs in size from that used in our former paper ${ }^{(6)}$.

The kinematics of this PMPMA can be expressed as follows ${ }^{(11)}$ :

$$
\boldsymbol{r}_{c}+\boldsymbol{R}_{e i}-\boldsymbol{r}_{b i}=L_{i} \boldsymbol{a}_{i}
$$

where $\boldsymbol{r}_{c}$ is the position vector from $O_{b}$ to $O_{e}, \boldsymbol{R}$ is the rotation matrix of the end coordinate system for the base coordinate system, $\boldsymbol{r}_{e i}$ is the position vector from $O_{e}$ to the $i$ th joint on the end part, $\boldsymbol{r}_{b i}$ is the position vector from $O_{b}$ to the $i$ th joint on the base part, $L_{i}$ is the length of the $i$ th actuator, and $\boldsymbol{a}_{i}$ is the directional vector of the $i$ th actuator $(i=1 \sim 3)$.

\subsection{PMPMA control system}

On the basis of the PMA control system in $\S 3$, we designed a system to enable the elbow joint angles of the PMPMA, $\theta$ and $\phi$, to be followed as references by controlling the contraction of each actuator, $l_{i}$. Figure 12 shows the overall structure of the control system. In this system, the reference contraction of each actuator, $l_{d i}$, is computed from the prescribed reference joint angles, $\theta_{r}$ and $\phi_{r}$, by means of an inverse kinematics equation derived from Eq. (14) and is then sent to the controller of each PMA. The PMA controllers are the same as that used in $\S 3$.

\subsection{Experimental results}

To investigate the performance of the tracking control of the proposed PMPMA system, we conducted experiments. In all the experiments, the three PMAs were pressurized initially up to the bias pressure, $p_{0}(=285 \mathrm{kPa})$. When the actuators were pressurized to $p_{0}$, the joint angles were set as 0 deg. A frequency response for the proposed control system with the second-order dynamic Preisach model is plotted with the symbol $\bigcirc$ in Fig. 13. Each value is the average of two experiments. This was obtained based on the time responses when a constant value for $\phi_{r}$ and various sinusoidal signals with an amplitude of $30 \mathrm{deg}$ and a time period from $1 \mathrm{~s}$ to $20 \mathrm{~s}$ for $\theta_{r}$ were inputted. For comparison, our previously 

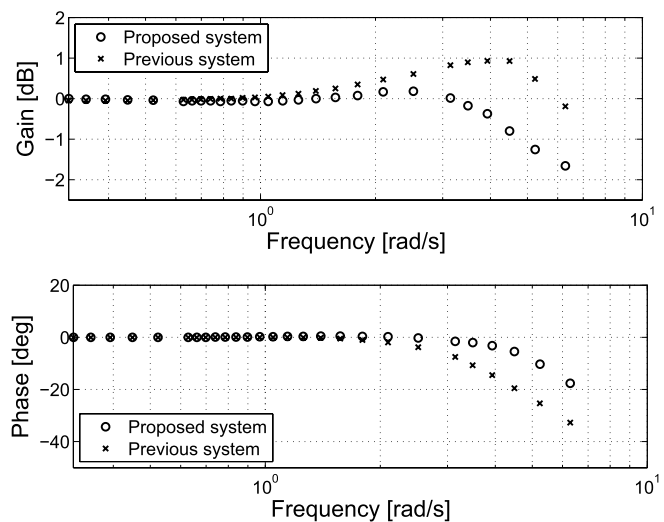

Fig. 13 Comparison of experimental frequency responses in the proposed and previous PMPMA control systems

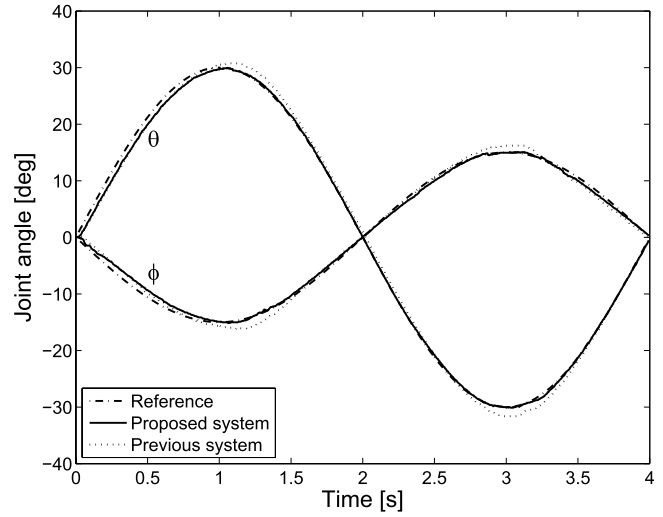

Fig. 14 Comparison of experimental time responses in the proposed and previous PMPMA control systems

proposed control system ${ }^{(6)}$ with the static Preisach model was also tested, and its result is plotted with the symbol $\times$ in Fig. 13. Time response examples when controlling $\theta$ and $\phi$ in the two systems are shown in Fig. 14. In this figure, the dashed-dotted, solid, and dotted lines indicate the references, responses with the proposed system, and responses with the previous system, respectively. These results demonstrate that the currently proposed technique is more effective even in the control of the PMPMA.

\section{Conclusion}

The present study was aimed at offering a technique to cope with some of the drawbacks of our previously proposed technique for modeling and controlling PMAs and to enhance the control performance. The results obtained in this study can be summarized as follows:

( 1 ) To avoid modeling errors due to the distortion of the hysteresis curve by the contracting and expanding speed in a PMA, we employed a dynamic Preisach model. We enabled the model to adjust its parameters on-line by applying recursive parameter estimation. Experimental comparisons showed that the modeled hysteresis agreed well with the measured one even when the PMA acted fast.

(2) Introducing the fast algorithm for finding the inverse of the static Preisach model enabled the real-time calculation of the hysteresis compensator outputs. This entirely eliminated the preliminary calculation for the hysteresis compensator.

( 3 ) Control systems incorporating the adaptive hysteresis compensator with the dynamic hysteresis model including the on-line tuned parameters and the fast inversion algorithm were constructed and examined in experiments for a single PMA and for a PMPMA. The proposed technique resulted in higher tracking performance for both of them than our previous technique, specifically in the high-speed range. 


\section{References}

( 1 ) Beater, P., Pneumatic Drives, (2007), Springer.

( 2 ) Kadota, K., Akai, M., Kawashima, K., and Kagawa, T., Development of Power-Assist Robot Arm using Pneumatic Rubber Muscles with a Balloon Sensor, Proceedings of the 18th IEEE International Symposium on Robot and Human Interactive Communication, (2009), pp. 546-551.

( 3 ) Jamwal, P. K., Xie, S., and Aw, K. C., Design Analysis of a Pneumatic Muscle Driven Wearable Parallel Robot for Ankle Joint Rehabilitation, Proceedings of 2010 IEEE/ASME International Conference on Mechatronics and Embedded Systems and Applications, (2010), pp. 403-408.

( 4 ) Kelasidi, E., Andrikopoulos, G., Nikolakopoulos, G., and Manesis, S., A survey on pneumatic muscle actuators modeling, Proceedings of 2011 IEEE International Symposium on Industrial Electronics, (2011), pp. 1263-1269.

( 5 ) Mayergoyz, I. D., Mathematical Models of Hysteresis and Their Applications, (2003), Elsevier.

( 6 ) Kosaki, T. and Sano, M., Control of a Parallel Manipulator Driven by Pneumatic Muscle Actuators Based on a Hysteresis Model, Journal of Environment and Engineering, Vol. 6, No. 2, (2011), pp. 316-327.

( 7 ) Iyer, R. V. and Tan, X., Control of Hysteretic Systems Through Inverse Compensation, IEEE Control Systems Magazine, Vol. 29, No. 1, (2009), pp. 83-99.

( 8 ) Ljung, L., System Identification, (1999), Prentice Hall.

( 9 ) Slock, D. T. M., On the Convergence Behavior of the LMS and the Normalized LMS Algorithms, IEEE Transactions on Signal Processing, Vol. 41, No. 9, (1993), pp. 28112825.

(10) Davino, D., Natale, C., Pirozzi, S., and Visone, C., A fast compensation algorithm for real-time control of magnetostrictive actuators, Journal of Magnetism and Magnetic Materials, (2005), pp. 1351-1354.

(11) Merlet, J.-P., Parallel Robots, (2006), Springer. 